\title{
Design of Optimal PID Controller Using NSGA-II Algorithm and Level Diagram
}

\author{
Amal MOHARAM, Mostafa A. El-HOSSEINI, Hesham A. ALI \\ Computers Engineering and Control systems Dept., Faculty of Engineering, \\ Mansura University, Egypt \\ eng.amalmoharam@yahoo.com, melhosseini@gmail.com, h_arafat_ali@mans.edu.eg
}

\begin{abstract}
This paper introduces a design for multi-objective PID controller using non-dominated sorting genetic algorithm (NSGA-II). When selecting the objectives to be optimized, it is taken into account to cover some important characteristics of the system like performance, robustness and control signals' smoothness. The decision making is done using Level diagram tool. Three tanks liquid level system control is discussed as a case study. The results show that this tool improves the process of decision making (DM). Also, comparisons with Ziegler and Nichols (Z-N) and different optimization methods are presented.
\end{abstract}

Keywords: Decision making (DM), Evolutionary algorithm (EA), Level Diagram (LD), Multi-objective optimization (MO), PID controller, Three Tanks Liquid Level System.

\section{Introduction}

In multi-objective optimization (MO), there is more than one objective to be optimized. Usually these objectives contradicts each other (i.e. optimize of one objective cannot be achieved without degradation of other objective). Hence there is no longer a single solution (as in a mono-objective optimization) but a group of trade-off solutions called Pareto points. The need for multiple Pareto points make evolutionary algorithms (EAs) more suitable for MO since the EAs work in parallel and can get more solutions in a single run[1,2].

EAs should be modified to be suitable for MO. This is due to the fact that it is required to have numerous solutions and thus need more diversity. Non-dominated sorting genetic algorithm (NSGA-II) [3] is considered one of efficient Multi-objective Optimization Evolutionary Algorithms (MOEAs). It is introduced in 2002 to overcome some shortcomings of NSGA [4]. Since then, it has proved its efficiency in many MO branches [5-8].

Decision making (DM) (i.e. selecting preferred solution points) is an important step in MO. Indeed, this is not an easy task since there are multiple trade-off solutions. Also, DM gets more complicated with the increase of number of objectives. To be able to carry out DM effectively, graphical presentation, can be helpful tool in our analysis. Scatter diagrams and Parallel coordinates [9, 10] are the most common graphical techniques used in MO analysis. However, these techniques lose clarity with increasing number of objectives. To overcome this difficulty, a new graphical technique called Level diagram (LD) [11] is introduced in 2008. Its idea is based on plotting each objective and decision variable in a separate sub-plot .These sub-plots are related to each other. This separation yields a good visibility for each objective and decision variable hence more capability on doing DM.

PID controller is the most widely used controller in industry because of its simplicity and robustness [12]. PID controller is still the perfect choice for many plants. However, finding the optimal parameters of PID controller is quite difficult especially in non-linear control system as in the liquid level control system. So, several methods have been proposed for tuning PID controller. One of these methods is Ziegler and Nichols method [13]. It is the oldest method and simplest one. Recently, many EAs such as genetic algorithm (GA) have been employed to tune PID controller in various plants [14-16].

For tuning PID controller, there are many different measures which can be used to compare the quality of controlled responses. These measures or objectives include time response specifications of the control system (i.e. overshoot, settling time...), integral performance indices and frequency domain objectives (i.e. sensitivity, complementary sensitivity...) [12, 17-19]. When designing a control system, these objectives should be selected carefully to represent demands of decision maker.

In this paper, due to its efficiency, NSGA-II is used for tuning MO-PID controller in three 
tanks liquid level system. For tuning PID controller, three objectives are selected to be minimized. The aim of selecting these objectives is trying to reach the best performance of the control system while keeping anti-disturbance ability and avoiding stress of the control actuator. The analysis of results is performed using a graphic LD tool.

The rest of the paper is organized as follows. Section 2 reviews related work. Section 3 describes a case study for tuning MO-PID controller in liquid level system. Section 4 analyzes the results using LD and comparisons held between NSGA-II and different optimization algorithms. Finally, section 5 concludes this paper.

\section{Related Work}

\subsection{Multi-objective terminology}

MO problem can be written in the form:

Minimize $J(x)=\left(J_{1}(x), J_{2}(x), J_{3}(x), \ldots, J_{k}(x)\right)$

for $k$ objective functions, $x \in R^{n}$

Subject to $m$ inequality constraints

$g_{i}(x) \leq 0 i=1,2, \ldots, m$,

and to $p$ equality constraints.

$h_{i}(x)=0 i=1,2, \ldots, p$,

where $x$ is defined as the decision vector, $J(x)$ as the objective vector.

Definition (Pareto Dominance):

Given a solution $x_{1}$ with objective vector $J\left(x_{1}\right)$, it dominates a second solution $x_{2}$ with objective vector $J\left(x_{2}\right)$ if and only if $J\left(x_{1}\right)$ is partially less than $J\left(x_{2}\right)$, i.e.

$\forall i \in[1,2, \ldots k], J_{i}\left(x_{1}\right) \leq J_{i}\left(x_{2}\right)$, and

$\exists q \in[1,2, \ldots . k], J_{q}\left(x_{1}\right)<J_{q}\left(x_{2}\right)$

Definition (Pareto optimality): A point $x$ is considered to be Pareto optimal if and only if there does not exist another point $\dot{x}$ so that $J(x)$ is dominated by $J(\dot{x})$. Pareto-optimal Set $P^{*}$ is considered to be the set that contains all of these solutions.

Definition (Pareto-front): The Pareto front $P F^{*}$ is defined as:

$$
P F^{*}=\left\{J(x)=\left(J_{1}(x), \ldots J_{k}(x)\right) \mid x \in P^{*}\right\}
$$

\subsection{Level diagram (LD)}

In addition to being synchronized with the objective and parameter diagram, classification of Pareto front points according to how much they are close to ideal points is the main key factor that reflects the performance of $\mathrm{LD}[11]$. The ideal point is the point that has the minimum value along the Pareto front for each objective. The steps of plotting LD are as follows:

1. For each objective, find its minimum and maximum values $\left\{J_{i}^{\min }=\min J_{i}(x)\right.$, $\left.J_{i}^{\max }=\max _{i}(x)\right\}$

2. Calculate the norm for each objective

$\bar{J}_{i}(x)=\frac{J_{i}(x)-J_{i}^{\min }}{J_{i}^{\max }-J_{i}^{\min }}$

3. Apply a norm (1-norm: $\left\|\bar{J}_{i}(x)\right\|_{1}$ or Euclidean norm: $\left\|\bar{J}_{i}(x)\right\|_{2}$ or $\infty$-norm: $\left.\left\|\bar{J}_{i}(x)\right\|_{\infty}\right)$ to evaluate the distance to the ideal point.

4. Pareto front point need to be ordered in ascending order of the value of the $\left\|\bar{J}_{i}(x)\right\|_{\text {norm }}$.

5. Plot a 2-D separate sub-plot for every objective and every decision variable. Axis $\mathrm{X}$ corresponds to values of the objective, or decision variables, in physical units. Axis $\mathrm{Y}$ represents the value of norm $\left\|\bar{J}_{i}(x)\right\|_{\text {norm }}$ in all sub-plots [11]. Thus a given solution will have the same Y-axis position in all sub-plots. This is very helpful in comparison among solutions.

Using $\infty$-norm in LD gives information concerning the worst objective for any point, and it is important in the trade-off analysis .So, this paper will use $\infty-$ norm in the analysis.

A comparison between 2-D classical plot and LD diagram, for simple MO test benchmark function with two objectives $J_{1}, J_{2}$ (convex, uniform Pareto Front), is shown in Figure 1 and Figure 2 respectively.

$$
J_{1}=\frac{1}{n} \sum_{i=1}^{n} x_{i}^{2}, \quad J_{2}=\frac{1}{n} \sum_{i=1}^{n}\left(x_{i}-2\right)^{2}, \quad 0 \leq x \leq 1
$$

From Figure 1 and Figure 2, we can see that, for example, point $A$ in 2-D classical can be represented by $(k+n)$ points in LD diagram $(k$ is the number of objectives and $n$ is the dimension of the decision vector $x$, i.e. $k$ points; 
for simplicity; are only shown in Figure 2, $n$ points are not shown). All the above mentioned points have the same value on the $\mathrm{Y}$ axis, and this position represents the distance to the ideal point related to specific norm.

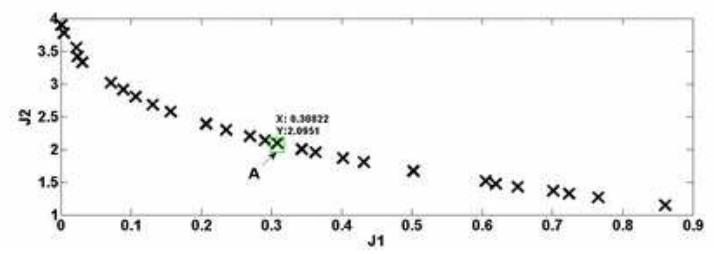

Figure 1. 2-D classical plot
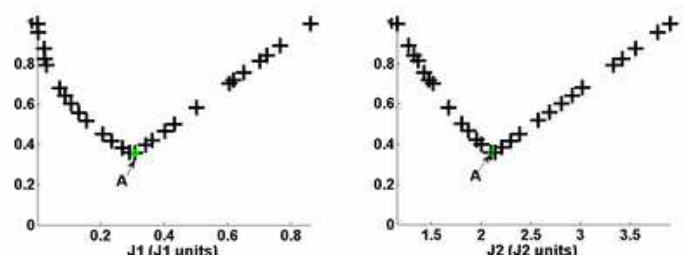

Figure 2. LD diagram \{norm- $\infty$ \} (the Pareto front only)

\subsection{NSGA-II}

Any EA, such as genetic algorithm, is based on three steps; selection, crossover and mutation. The key design of any MO genetic algorithm is selection step [20]. Crossover and mutation steps always remain as usual. The steps of selection procedure in NSGA-II can be summarized as follows:

- Sort the population based on domination concept into fronts; each front is dominated only by the previous fronts. The sorting is done using new fast technique to reduce the high computational complexity used in NSGA.

- Calculate the crowding distance for each individual in each front. The crowding distance is an estimation about the crowding of the region surrounded by the individual by calculating how far the individual from its neighbors.

- Select the population for next generation. This is based on two factors; the rank of the individual in any front and its crowding distance. For example, as shown in Figure 3, select first the individuals in the first and second fronts (i.e. they have the advanced rank). Then compare among individuals in the third front according to their crowding distance (i.e. Crowding distance is compared only among the individuals in the same front). Select the individuals with less crowding distance (i.e. to keep diversity) until complete the size of population.

As shown in Figure 3, one important feature of NSGA-II, compared to NSGA, is using elitist to avoid loss of good solutions once they are found.

\section{Case Study: Three tanks Liquid level control}

Liquid level control is very important problem in many industrial applications as in water purification systems, industrial chemical processing and boilers in all the industries. In

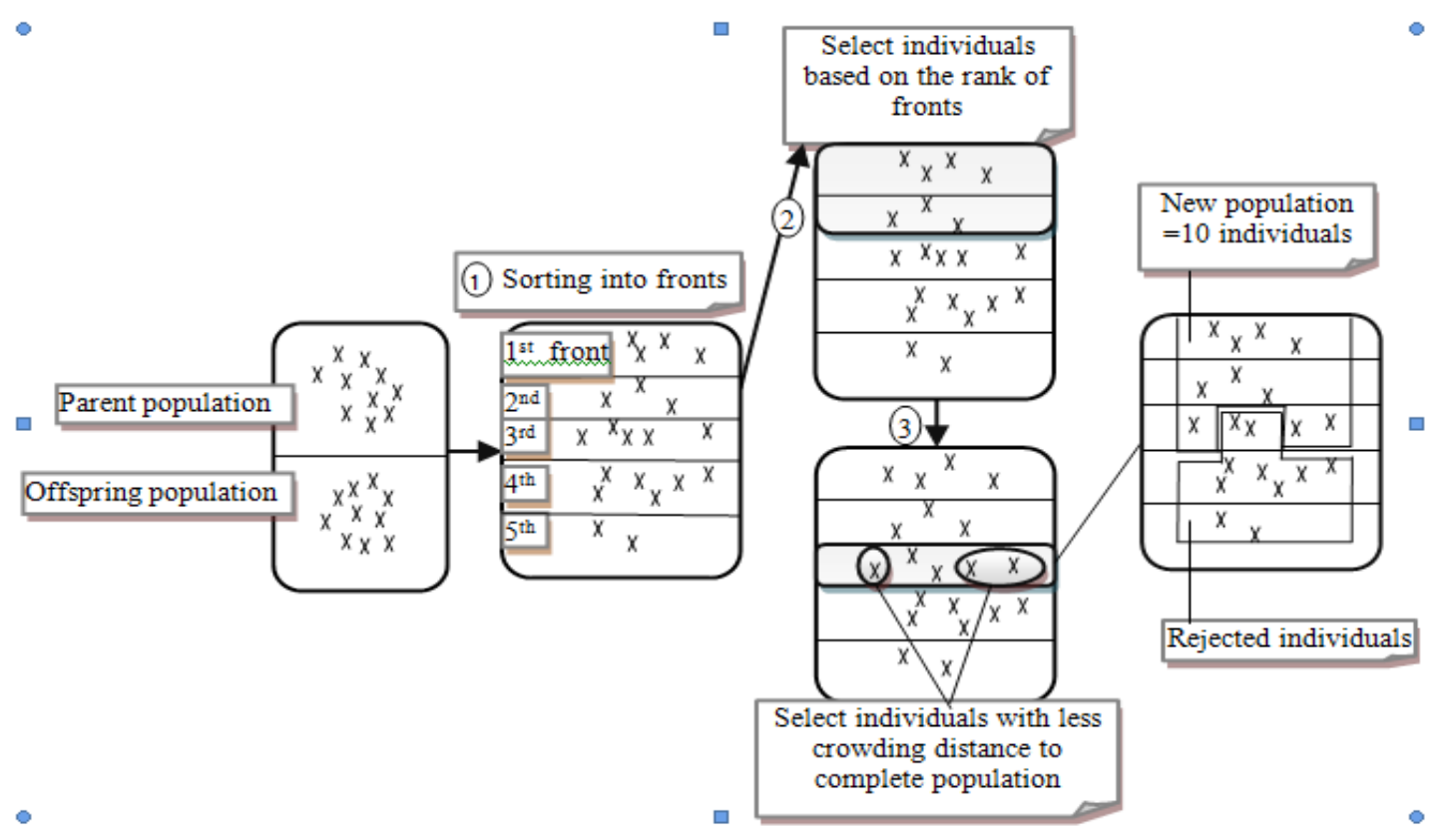

Figure 3. Selection procedure in NSGA-II for population=10 individuals 
the process industries, liquids require to be pumped, stored in tanks, and then pumped to another tank. The liquid will be processed by chemical and mixed treatment in the tanks. So, tanks are always found in groups and three tanks liquid level system is considered one of the widely known applications.

\subsection{Modelling of three tanks liquid level system [19,21]}

A simple structure of the three tanks liquid level system is shown in Figure 4.

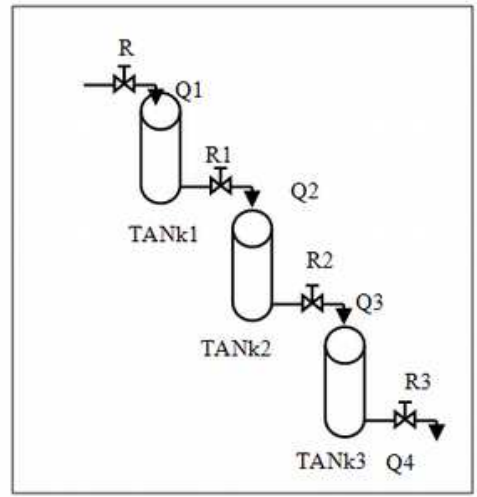

Figure 4. Simple structure of the three tanks liquid level system

According to mass balance:

$Q_{i}(t)-Q_{i+1}(t)=A d h_{i} / d t$

$Q_{i+1}=$ constant $\times \sqrt{h_{i}}$

From equation (2), it can be seen that the water level is a system with nonlinearity. Around the equilibrium point, equation (2) can be rewritten approximately as:

$$
Q_{i+1} \approx \frac{1}{R_{i}} \times h_{i}
$$

where $Q_{i}, Q_{i+1}$ denote the flow rate of liquid into and out of the tank; respectively ( $i$ is the number of the tank; $i=1,2,3 . A$ is the cross sectional area of the tank, $h_{i}$ is the liquid level of the tank. $R_{i}$ is the linear resistance of tank.

Substitute $Q_{i+1}$ in equation (1) by its value in equation (3) and calculate the Laplace Transformation of equation (1):

$$
G(s)=\frac{H_{i}(s)}{Q_{i}(s)}=\frac{R_{i}}{R_{i} A s+1}
$$

Thus, the overall transfer function of the three tanks system is:

$$
\frac{H_{3}(s)}{Q_{1}(s)}=\frac{H_{3}(s)}{Q_{3}(s)} \times \frac{H_{2}(s)}{Q_{2}(s)} \times \frac{H_{1}(s)}{Q_{1}(s)} \times \frac{Q_{3} Q_{2}}{h_{2} h_{1}}
$$

Substitute the equation (3) and equation (4) into equation (5):

$\frac{H_{3}(s)}{Q_{1}(s)}=\left(\frac{R_{1}}{A_{1} R_{1} s+1}\right)\left(\frac{R_{2}}{A_{2} R_{2} s+1}\right)\left(\frac{R_{3}}{A_{3} R_{3} s+1}\right) \times \frac{1}{R_{1} R_{2}}$

\subsection{Block diagram of three tanks liquid level system}

For $A_{1}=A_{2}=1.5, A_{3}=1, R_{1}=R_{2}=2, R_{3}=3$, the block diagram of three tanks liquid level system is shown in Figure 5.

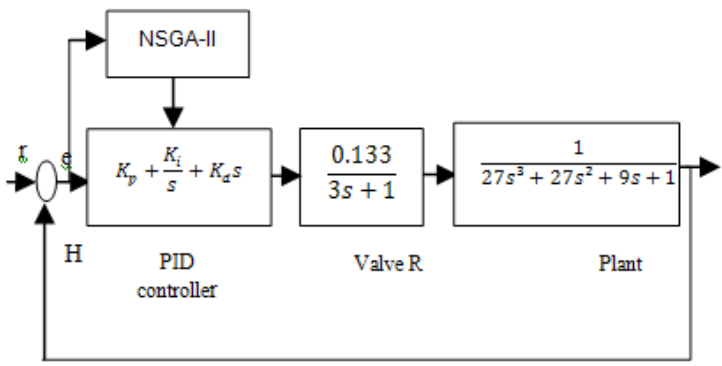

Figure 5. Block diagram of three tanks liquid level system with PID controller

\section{Settings and Results}

\subsection{Settings}

The decision vector $=\left[K_{d} K_{p} K_{i}\right]$;

$K p, K_{i}, K_{d}$ are proportional gain, integral gain and derivative gain; of PID controller; respectively.

$$
\begin{aligned}
& x_{\text {minimum }}=\left[\begin{array}{lll}
0 & 0 & 0
\end{array}\right] ; \\
& x_{\text {maximum }}=\left[\begin{array}{lll}
20 & 10 & 1
\end{array}\right] .
\end{aligned}
$$

The objectives to be minimized

Min $J=\left[\begin{array}{lll}J_{1} & J_{2} & J_{3}\end{array}\right]$. Table 1 shows the details of these objectives.

Table 1. The objectives to be minimized

\begin{tabular}{|c|c|c|}
\hline$J_{1}$ & $\begin{array}{l}\text { Integral r of } \\
\text { Absolute Error } \\
\text { value ( IAE ) }\end{array}$ & $I A E=\int_{t_{0}}^{t_{f}}|r| t \mid-y(t) \vee d t$ \\
\hline$J_{2}$ & $\begin{array}{l}\text { Total variation } \\
\text { of control action } \\
(T V)\end{array}$ & $T V=\int_{t_{0}}^{t_{f}}\left|\frac{d u}{d t}\right|$ \\
\hline$J_{3}$ & $\begin{array}{l}\text { Maximum value } \\
\text { of Sensitivity } \\
\text { function (Ms) }\end{array}$ & $M s=\left\|(I(s)+P(s) C(s))^{-1}\right\|_{\infty}$ \\
\hline
\end{tabular}

where $r, y \wedge u$ are the set point signal, the actual value of the process and the control variable, respectively. $P(s)$ and $C(s)$ are the process transfer function and controller transfer function respectively. The selected objectives are a trade-off performance, control signals' 
smoothness (i.e. to avoid stress of the control actuator) and robustness. The parameters of NSGA-II that used are crossover ratio $=0.9$, mutation ratio $=0.1$, and maximum number of function evaluations $(\mathrm{FEs})=10000$.

\subsection{Results and comparison}

\subsubsection{Remarks on results}

Parallel coordinates plot and scatter plot of Pareto front are shown in Figure 6 and Figure 7 respectively. From Figure 6, we can see that the main shortcoming of the parallel coordinates plot is its clutter. Scatter plot is extremely vague, has limited observations and hence the analysis is very difficult. In this paper, the analysis of the results is carried out using LD visualization tool [23]. By using it, decision maker can select, classify and remove points in the Pareto front/set easily. LD of the Pareto front/set results is shown in Figure 8. From Figure 8, we can see:

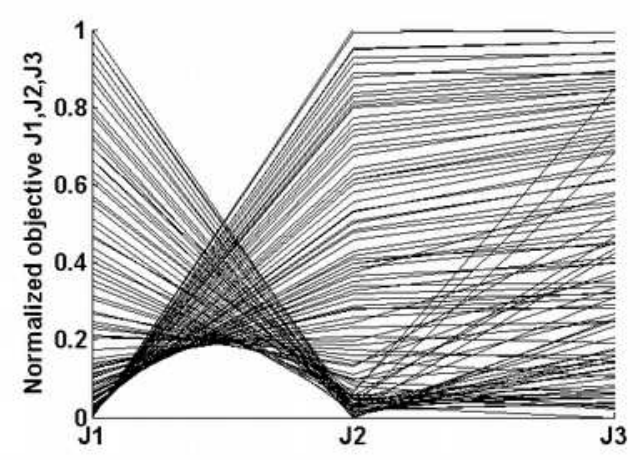

Figure 6. Parallel coordinate for Pareto front
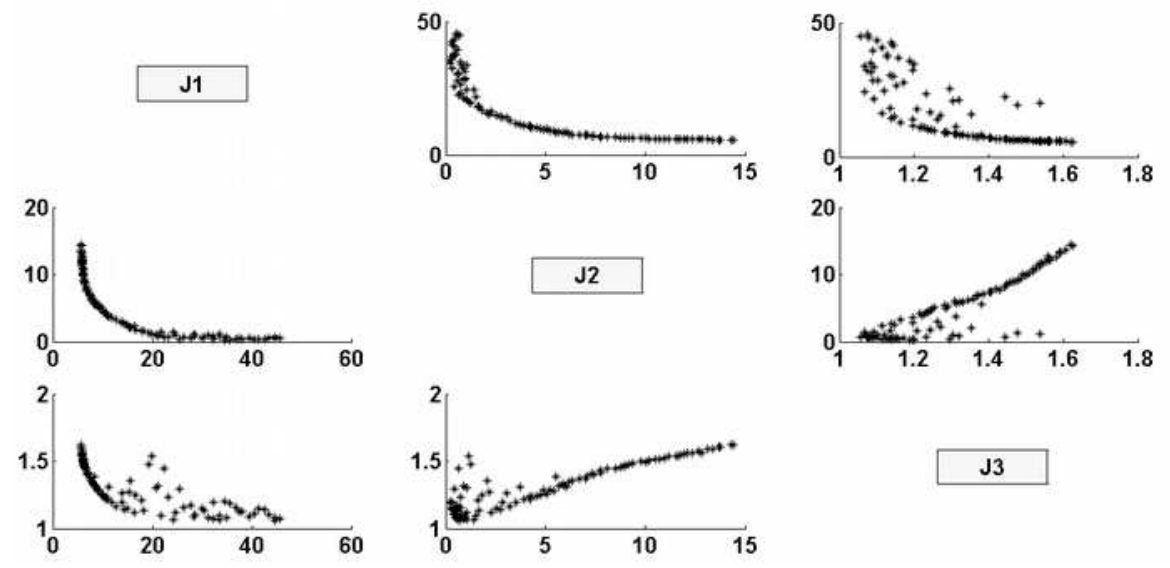

Figure 7. Scatter plot for Pareto front

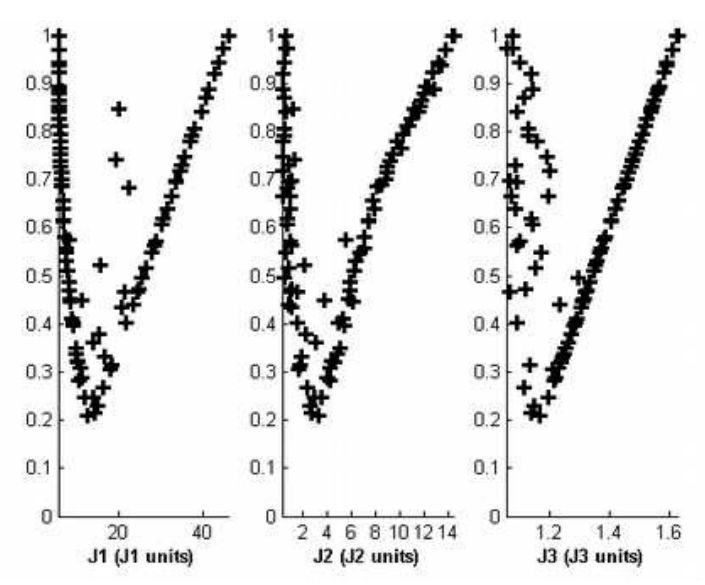

Figure 8.A. Pareto Front LD

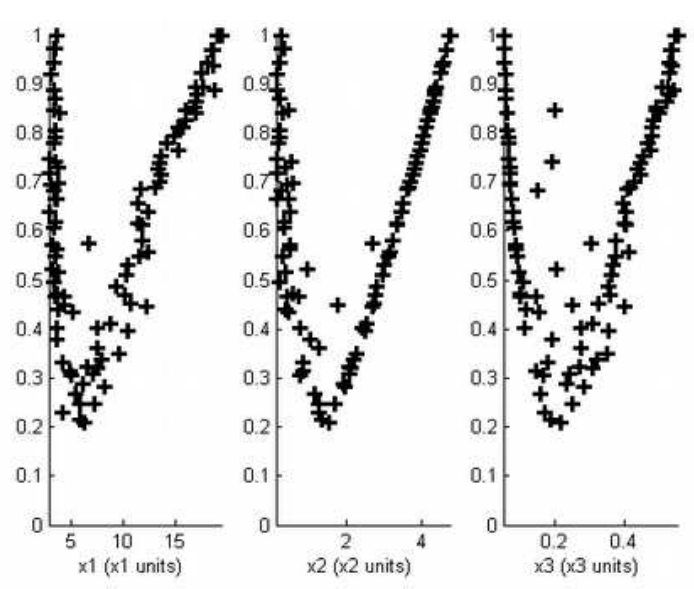

Figure 8.B. Pareto Set LD 
1. There are no discontinuities in any objectives, which means that the decision maker can easily choose the point that agreed with his preferences.

2. Numerous points are found at bottom level of Pareto front (i.e. close to the ideal point). So, it is easy to choose a point that is a trade off all objectives. The best point (unbiased to any objective; the closet to ideal point) is $J=[12.75 ; 3.21 ; 1.16]$, $x=[6.26 ; 1.53,0.22]$.

3. Improving the performance (i.e. decreasing IAE) cannot be done without increasing the total variation of control action ( $T V$ ); this is shown clearly in Figure 9 (In this figure, classifying points with IAE less than 10).

4. Decreasing sensitivity cannot be done without affecting on the performance of the system; this is shown in Figure 10 (In this figure, classifying points with sensitivity less than 1.2).

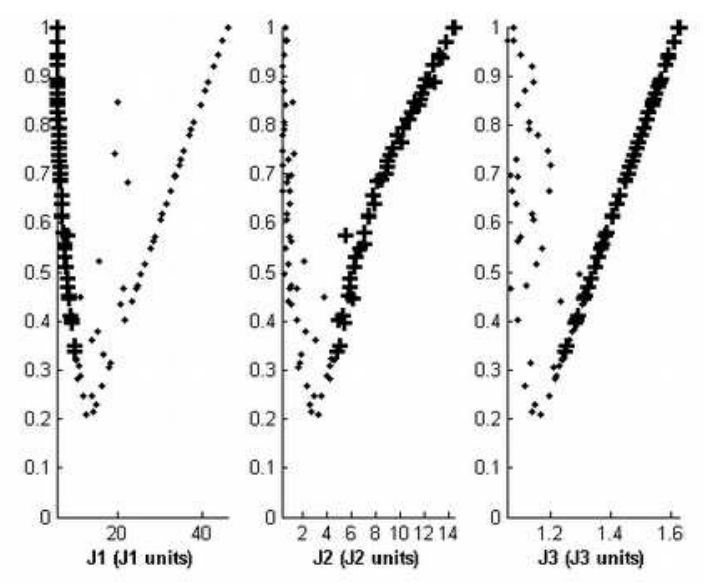

Figure 9. Pareto Front LD (classifying points with $\mathrm{IAE}<10$ )
5. In the Pareto set, there are no discontinuities. Also, the Pareto set shows that $x_{2}, x_{3}$ are localized between $[0,5],[0$, $0.5]$ respectively; this means that we can change the bound of the search for $x_{2}, x_{3}$.

\subsubsection{Comparison}

NSGA-II is compared against four optimization methods (i.e. Box method [24], Random search algorithm, MO genetic algorithm(MOGA) [25], MO Dragonfly algorithm(MODA) [26]) for the same number of FEs (i.e. FEs $=10000$ ). From analysis of these results, it is found that Random search solutions can dominate 2 solutions of NSGA-II. The other three algorithms results dominate 1 solution of NSGA-II. Table 2 shows the comparison results of competitive algorithms (i.e. the nondominated points), Ziegler-Nichols (Z-N) [13] and NSGA-II algorithm.

As can be depicted from Table 2, Z-N gives the maximum overshoot. This is the main

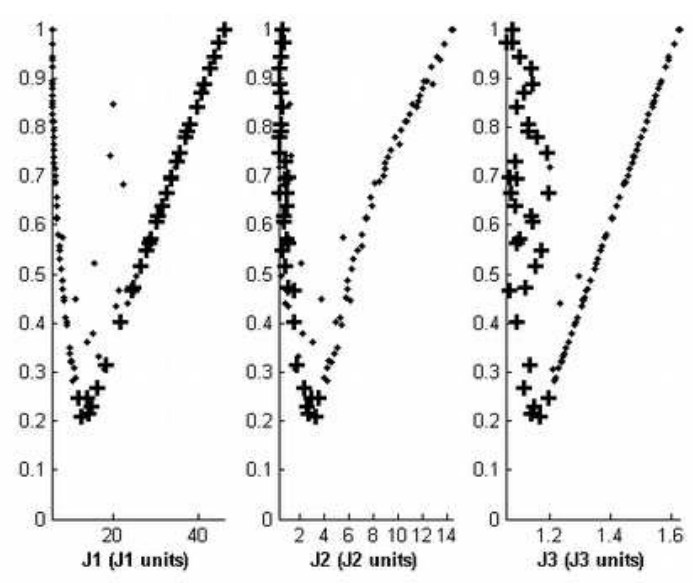

Figure 10. Pareto Front LD (classifying points with $M s<1.2$ )

Table 2. Comparison using different controllers

\begin{tabular}{|c|c|c|c|c|c|c|c|}
\hline & {$[k d k p k i]$} & $I A E$ & $T V$ & $M s$ & overshoot & Settling time & Rise time \\
\hline Z-N & {$[18.98 ; 7.4 ; 0.72]$} & 7.74 & 29.06 & 2.3 & 31.4 & 39.3 & 4.17 \\
\hline Box method & {$[6.12 ; 1.266 ; 0.247]$} & 13.74 & 2.89 & 1.23 & 8.8703 & 53.8292 & 17.5388 \\
\hline $\begin{array}{c}\text { Random search } \\
\text { algorithm (2 } \\
\text { nondominated points) }\end{array}$ & {$[6.81 ; 1.26 ; 0.20]$} & 14.2 & 2.7 & 1.3 & 4.13 & 59.48 & 22.28 \\
\cline { 2 - 9 } & {$[6.9 ; 1.3 ; 0.22]$} & 3.78 & 2.8 & 1.14 & 5.67 & 57.69 & 20.29 \\
\hline MO genetic algorithm & {$[3.716 ; 1.256 ; 0.198]$} & 13.86 & 2.63 & 1.22 & 2.8715 & 49.8 & 19.418 \\
\hline Dragon algorithm & {$[8.705 ; 2.446 ; 0.31]$} & 9.1005 & 5.1336 & 1.2806 & 2.9787 & 39.5689 & 12.4145 \\
\hline $\begin{array}{c}\text { NSGA-II (the best trade- } \\
\text { off point) }\end{array}$ & {$[6.26 ; 1.53,0.22]$} & 12.75 & 3.21 & 1.16 & 2.71 & 50.66 & 20 \\
\hline $\begin{array}{c}\text { NSGA-II (minimum } \\
I A E)\end{array}$ & {$[19.32 ; 4.73 ; 0.54]$} & 5.71 & 14.39 & 1.62 & 5.2 & 30.97 & 5.37 \\
\hline NSGA-II (minimum $T V$ ) & {$[2.82 ; 0.091 ; 0.073]$} & 34.47 & 0.25 & 1.2 & 0.0247 & 87.0481 & 56.2312 \\
\hline NSGA-II (minimum $M s)$ & {$[3.48 ; 0.318 ; 0.055]$} & 44.66 & 0.6815 & 1.055 & 0 & 127.17 & 83.2934 \\
\hline
\end{tabular}


drawback of Z-N that make researchers search for other efficient algorithms [22].Random search can get two non-dominated points which indeed are very close to each other. MOGA gets discriminated better solution which is very close to the best trade-off point of NSGA-II.

Figure 11 shows the step response of three tanks liquid level system for some nondominated points. From figure 11, we can see that the solution of MODA has good step response characteristics. NSGA-II can get various results that can satisfy most demands of decision maker. For example, if decision maker is concerned in improving the performance of the system, he can select NSGA-II point which achieve minimum $I A E$. This point surely has the maximum $T V$ and $M s$.

\section{Conclusion}

This paper presents a design of MO-PID controller in three tanks liquid level system using NSGA-II algorithm and LD tool. The main objectives to be minimized are selected to achieve high performance of the system and high robustness without affecting the life span of the control valve. It is clear from the results that NSGA-II, compared to other algorithms, can obtain various solutions that can satisfy different demands of decision maker. For future work, it is desired to reformulate the design problem to incorporate other objectives function.

\section{REFERENCES}

1. MIETTINEN, K. M., Nonlinear Multiobjective Optimization, Intl. Series in Operation Research and Management Science, Kluwer Academic, 1998.

2. ABRAHAM, A., L. JAIN, R. GOLDBERG, Evolutionary

Multi-objective

Optimization Theoretical Advances and Applications, Springer, 2005.

3. DEB, K., A. PRATAP, S. AGARWALAND, T. MEYARIVAN, A Fast and Elitist Multi-objective Genetic Algorithm: NSGA-II., IEEE Transaction on Evolutionary Computation, vol. 6, no. 2, 2002, pp. 182-197.

4. SRINIVAS, N., K. DEB, Multi-objective Optimization using Nondominated Sorting in Genetic Algorithms, Evolutionary Computing, vol. 2, no. 3, 1994, pp. 221-248.

5. XIANG-ZHONG, G., Multi-objective PID Controller Based on NSGA-II Algorithm with Application to Main Steam Temperature Control, International Conference on Artificial Intelligence and Computer Intelligence, AICI '09, Shanghai, 2009, vol. 4, pp. 21-25.

6. PIRES, D. F., C. H. ANTUNES, A. G. MARTINS, NSGA-II with Local Search for a Multi-objective Reactive Power

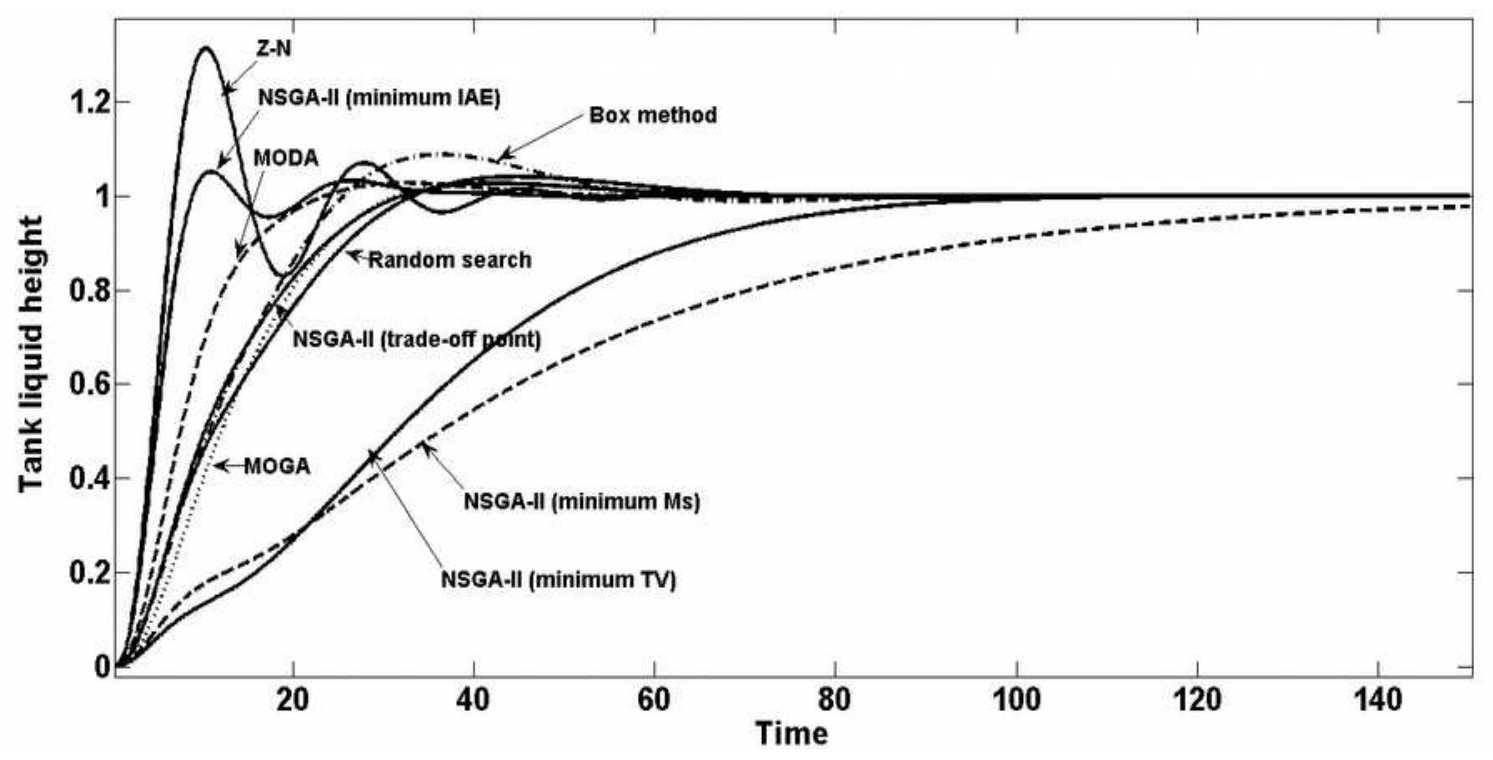

Figure 11. Step response of three tanks liquid level system with different multi-objective controllers 
Compensation Problem, International, Journal of Electrical Power \& Energy Systems, vol. 43, no. 1, 2012, pp. 313-324.

7. KALAIVANI, L., P. SUBBURAJ, M. WILLJUICE IRUTHAYARAJAN, Speed Control of Switched Reluctance Motor with Torque Ripple Reduction using Non-dominated Sorting Genetic Algorithm (NSGA-II), International Journal of Electrical Power \& Energy Systems, vol. 53, 2013, pp. 69-77.

8. KASHYAP, R., D. P. VIDYARTHI, Security Driven Scheduling Model for Computational Grid Using NSGA-II, Journal of Grid Computing, vol. 11, no. 4, 2013, pp. 721-734.

9. MARCH, P., T. HOLLAND, Graphics and GUIs with MATLAB, Chapman \& Hall/CRC, 2003.

10. INSELBERG, A., The Plane with Parallel Coordinates, Visual Computing, vol. 1, 1985, pp. 69-91.

11. BLASCO, X., J. HERRERO, J. SANCHIS, M. MARTÍNEZ, A New Graphical Visualization of n-Dimensional Pareto Front for Decision-making in Multiobjective Optimization, Information Sciences, vol. 178(20), 2008, pp. 3908-3924.

12. ÅSTRÖM, K., T. HÄGGLUND, Advanced PID Control, ISA, 2005.

13. ZIEGLER, J. G., N. B. NICHOLS, Optimum Settings for Automatic Controllers, ASME Transactions, 1942, pp. 759-768.

14. JINGI, W., X. YANG, C. LEI, The Application of GA-based PID Parameter Optimization for the Control of Superheated Steam Temperature, International Conference on Machine Learning and Cybernetics (ICMLC), 2012.

15. AYALA, H., L. COELHO, Tuning of PID Controller based on a Multi-objective Genetic Algorithm Applied to a Robotic Manipulator, Expert Systems with Applications, vol. 39(10), 2012, pp. 8968-8974.

16. ÜNAL, M., A. AK, V. TOPUZ, H. ERDAL, Optimization of PID Controllers Using Ant Colony and Genetic Algorithms, Studies in Computational Intelligence, vol. 449, Springer, 2013.
17. OGATA, K., Modern control Engineering, Prentice Hall, 1990.

18. REYNOSO-MEZA, G., S. GARCÍANIETO, J. SANCHIS, F. X. BLASCO, Controller Tuning by Means of MultiObjective Optimization Algorithms: A Global Tuning Framework, IEEE Transactions on Control Systems Technology, vol. 21(2), 2013, pp. 445-458.

19. WADE, H. L., Basic and Advanced Regulatory Control: System Design and Application, 2nd Edition, ISA, 2004.

20. El-SEHIEMY, R. A., M. A. El-HOSSEINI, A. HASSANIEN, Multi-objective RealCoded Genetic Algorithm for Economic/ Environmental Dispatch Problem, Studies in Informatics and Control, vol. 22 , no. 2 , 2013, pp. 113-122.

21. KATAL, N., S. KR. SINGH, A. TRIPATHI, S. G. MODANI, Optimizing the Response of a PID Controller for Three Tank Liquid Level System using Multiobjective Genetic Algorithm, Proceedings of the International Conference on Advance in Electronics, Electrical and Computer Science Engineering, EEC 2012, pp. 40-43.

22. VISIOLI, A., Fuzzy Logic Based SetPoint Weight Tuning of PID Controllers, IEEE Transactions on Systems, Man and Cybernetics - Part A: Systems and Humans, vol. 29 , no. 6,1999 , pp. 587-592.

23. http://www.mathworks.com/matlabcentral/f ileexchange/24042

24. BOX, M. J., A New Method of Constrained Optimization and a Comparison with Other Methods, Computer Journal, vol. 8(1), 1965, pp. 42-52.

25. http://www.mathworks.com/help/gads/inde x.html

26. MIRJALILI, S., Dragonfly Algorithm: A New Meta-heuristic Optimization Technique for Solving Single-objective, Discrete, and Multi-objective Problems, Neural Computing and Applications, Springer, 2015. 